\title{
Innovación en las empresas agroexportadoras de la región Junín 2017
}

\section{Innovation in agro-export companies in the Junín region 2017}

\section{'Gago Inga, E.T.; Ccencho Parí, G.}

\author{
Facultad de Economía, Universidad Nacional del Centro del Perú
}

Email: ccenchop28@hotmail.com

\section{Resumen}

La globalización impone nuevos retos a las empresas dedicadas a la agroexportación, pues la tecnología establece nuevos métodos de producción de acorde al mercado con nuevas técnicas y estrategias que vinculan a la innovación de las empresas. Este estudio determina la influencia de las innovaciones del producto, de procesos, de organización y de mercado (marketing) en las ventas de las empresas agroexportadoras de la región Junín. Para que una empresa se mantenga en el mercado, es necesario estar a la vanguardia, innovando el producto como tal, con nuevas características y detalles; así también, en el proceso productivo, de manera que se puedan reducir los costos de producción implementando innovadores métodos de producción.

Para el desarrollo de la investigación, se procedió a aplicar un cuestionario a una muestra de 36 empresas agroexportadoras de la región Junín; asimismo, se desarrolló un modelo econométrico para visualizar la influencia de la innovación en las ventas exportadas. Los resultados señalan que el modelo planteado muestra que la innovación explica significativamente (sig. $<0.05)$ a las agroexportaciones, específicamente, las innovaciones de productos, de procesos y de mercado $(\mathrm{R}$-cuadrado=0.80; F-stat=42.76). Por otra parte, la innovación organizativa no explica los niveles de venta de exportaciones, pero estaría incluida dentro de los otros tipos de innovación, teniendo en consideración que una buena organización permite contar con buenas presentaciones de productos, adecuados procesos y estrategias de mercado.

Palabras clave: agroexportación, empresa, innovación, estrategias de mercado

\begin{abstract}
Globalization imposes new challenges on companies engaged in agro-exporting, because technology establishes new production methods according to the market with new techniques and strategies that link to innovation of companies. This study determines the influence of product, process, organization and market (marketing) innovations on the sales of agro-exporting companies in the Junin region. For a company to remain in the market it is necessary to be at the vanguard, innovating the product as such with new features and details, as well as in the production process so that production costs can be reduced by implementing innovative production methods.

For the development of the research, a questionnaire was applied to a sample of 36 agro-exporting companies in the Junin region. Likewise, an econometric model was developed to visualize the influence of innovation on exported sales. Results indicate that the proposed model shows that the proposed model shows that innovation significantly explains (sig. < 0.05) agro-exports, specifically, product, process and market innovations (R-squared $=0.80$, F-stat $=42.76)$. On the other hand, organizational innovation does not explain export sales levels, but it would be included in other types of innovation, taking into account that a good organization allows good product presentations, appropriate processes and market strategies.
\end{abstract}

Keywords: agroexportation, companies, innovation, marketing strategies 


\section{Introducción}

La innovación es una necesidad para la generación de valor empresarial, debido a que representa el primordial fundamento de crecimiento en productividad y la producción (Albizu, Olazaran, Otero \& Lavía, 2012; Turiján, Damián, Ramírez, Juárez \& Estrella, 2012; y, Hudec \& Prochadzkova, 2015), innovar es generar valor basado en el cambio de conocimientos.

Flores, Del y Flores (2013) y Olaya (2008), señalan que al elevar el valor de la empresa mejora la cuenta de resultados, tanto en imagen como posición de la empresa en el mercado, debido a un mejor comportamiento de su oferta en el mercado. La innovación está vinculado a un contexto netamente tecnológico e industrial (Berk, 2018; Kleinsmann, Valkenburg \& Sluijs, 2017; Sánchez, Escobar, Sánchez \& Concha, 2013; Tello, 2017).

La innovación es un factor importante, puesto que tiene varias implicancias especialmente en desenvolvimiento empresarial, lo cual permite avanzar en el campo productivo, comercial y organizacional (Gras \& Hernández, 2016; Hernández, Mirafuentes \& Santamaría, 2016). Ante esto, la innovación está en función a tres principales fundamentos, al cambio como una vía, al valor como el objetivo y al conocimiento como la base de todo (Bin, et al., 2013; Bin \& Salles-Filhoa, 2012; Von Mühlen, et al., 2016).

El principal factor desconocido es que, en muchos casos empresariales, no se está entendiendo los efectos de la innovación para la mejora de su accionar (Carvalho, Avellar, Carvalho \& Avellar, 2017; Prim, Amal \& Carvalho, 2016). Debido a la ausencia de estudios relacionados al tema, se desconoce las nuevas formas de producción, de procesos, de organización y marketing que las empresas agroexportadoras de la región Junín están utilizando para seguir creciendo en el mercado.

Aunque se viene gestionando el Programa Nacional de Innovación en las empresas agroexportadoras, aún falta promoción e impulso por parte del Estado a diversas empresas en el Perú, toda vez, que no cuentan con la capacidad innovativa para alcanzar un nivel de exportación apropiado (PromPerú, 2017). En el caso de las empresas de la región Junín, estas continúan actuando con esquemas tradicionales; es decir, no están innovando. El informe desarrollado para la región Junín por parte de la Comisión de Promoción del Perú para la Exportación y el Turismo (PromPerú, 2017), señala que se encuentran registrados formalmente 166 empresas exportadoras ubicadas en la sierra y selva; no obstante, solo 86 empresas se dedican a la agroexportación, las cuales aplican esquemas poco innovadores que no contribuyen con el desarrollo de ventajas competitivas.

El objetivo de esta investigación consiste en determinar la influencia de la innovación, en cada uno de sus tipos, en las ventas de las empresas agroexportadoras de la región Junín. Los tipos de innovación establecidos en el Manual de Oslo son: la innovación de producto, la innovación de proceso, la innovación en marketing (o de mercado), y la innovación en organización (Enjolras, Camargo \& Schmitt, 2016).

La innovación de productos dentro de una empresa agroexportadora, debe entenderse como las presentaciones que realiza la empresa, así como las propiedades inherentes en ella, tales como la forma, color, sabor, empaque, tamaño y, principalmente, calidad (Chávez, Lombeida, Pazmiño \& Flora del C, 2015; Scoponi, Días, Pesce, Schmidt \& Gzain, 2016). Respecto a la innovación de procesos, esta consiste en la adaptación de técnicas y tecnologías, así como las certificaciones nacionales e internacionales para la producción agrícola (Rosario, Santa Rita \& de Albuquerque, 2013; Sánchez-Mejía \& Gutiérrez-Terán, 2013; Vargas-Canales, Palacios-Rangel, Camacho-Vera, Aguilar-Ávila \& Ocampo-Ledesma, 2015), tales como los certificados de SENASA (certificado sanitario), ISO 22000 (inocuidad alimentaria), certificado fitosanitario y otros, que los países demandantes requieren para garantizar el consumo de los productos agrícolas.

La innovación de la organización, dentro de una empresa agroexportadora, debe contener criterios de profesionalismo y especialización dentro del proceso productivo y de la estructura orgánica (Avendaño-Ruiz, 2017; Barrientos-Fuentes \& Berg, 2013). Por ejemplo, es indispensable que el recurso humano sea un factor que se encuentre capacitado, por lo que es recomendable contar con profesionales y técnicos que se encarguen de la supervisión y el desarrollo de los procesos; además, se requieren profesionales aptos para administrar y gerenciar a la empresa agroexportadora, de preferencia con experiencia en el segmento de las exportaciones (Fadhil, Syamsul Maarif, Bantacut \& Hermawan, 2017; Fuentes \& Soto, 2015; Vargas-Canales, et al., 2015). Y la innovación en marketing o de mercado, representa la promoción y publicidad que realiza una empresa agroexportadora para dar a conocer las propiedades de sus productos, de preferencia la publicidad puede realizarse vía web de manera que los importadores de los países demandantes tengan conocimientos de los productos agrícolas (Frezatti, Bido, Cruz \& Machado, 2017; Fuentes \& Soto, 2015).

Esta investigación es un referente para el desarrollo de investigaciones asociadas a la innovación, de manera que se verifique si el nivel o tipo de innovación que aplican influye en la producción, ventas e ingresos de empresas. Asimismo, se ha tomando el Manual de Oslo para identificar los tipos de innovación que vienen aplicando las empresas y su respectivo avance. 


\section{Materiales y métodos}

La investigación se efectuó en las empresas agroexportadoras de la región Junín, cuya población fue de 86 empresas, distribuidas en las áreas naturales de sierra y selva y que se dedican a la exportación de café, cacao, palta y otras frutas. Teniendo en cuenta que el estudio se asocia con la innovación, se consideraron dentro de la muestra, a empresas que hayan aplicado algún tipo de innovación y que se encuentran operativas en el 2017; descartándose a aquellas empresas cuyas oficinas principales fueron localizadas fuera del área de estudio. Es así como se estimó un tamaño de muestra de 36 empresas agroexportadoras.

El diseño de investigación fue no experimental transversal explicativo, teniendo en cuenta que se ha determinado la influencia de la innovación en las ventas de las empresas agroexportadoras en estudio. Asimismo, para la recopilación de los datos, se empleó la técnica de la encuesta y como instrumento un cuestionario, el cual fue desarrollado por los investigadores cuyo objetivo fue obtener información respecto a los tipos de innovación que desarrollaron las empresas, así como el nivel de ventas registradas en el año de estudio. Los cuestionarios se entregaron a los responsables de cada empresa (gerente, director, y administrador) para que ellos los puedan responder en un tiempo establecido de 10 minutos. La aplicación de las encuestas no fueron anónimas, ya que se requería saber su procedencia.

\section{Pruebas estadísticas}

Para la ejecución de la investigación se procedió a describir el mercado de las empresas agroexportadoras de la región Junín y desarrollar un modelo econométrico que expresó las ventas de las empresas agroexportadoras frente a los tipos de innovaciones realizadas en las empresas agroexportadoras durante el año 2017. Para el desarrollo del modelo se han tomado un indicador representativo por cada variable de estudio: para la variable innovación del producto, se tomó el indicador de presentaciones de los productos (X1); para la variable innovación de procesos, el indicador certificaciones (X2); para la variable innovación organizativa, el indicador porcentaje de personal con estudios superiores (X3) y; para la variable innovación de mercado, el indicador aplicación publicidad (X4). En cuanto a la variable ventas, se tomó el indicador de ingresos totales por exportación (Y) durante el año 2017 y residuos $(\mu)$, que explica a otras variables. Siendo el modelo econométrico el siguiente:

$\mathrm{Y}=\exp (\beta 0+\beta 1 \mathrm{X} 1+\beta 2 \mathrm{X} 2+\beta 3 \mathrm{X} 3+\beta 4 \mathrm{X} 4+\mu)$

Para el procesamiento de los datos se empleó el Software Eviews ${ }^{\circledR}$ 10, dado que con el uso del programa resulta posible verificar el cumplimiento de los supuestos (homocedasticidad, independencia de las variables explicativas y normalidad de residuos) de un modelo log de regresión multiple con corte transversal aplicando Mínimos Cuadrados Ordinarios (MICO). Es así que, se verificó la bondad de ajuste ( $\mathrm{R}$ - Cuadrado), el nivel de significancia individual (t-stat) y global (F-stat) y el criterio de información de Akaike. Asimismo, se aplicó el test de redundancia de variable para verificar que las variables sean representativas y explicativas.

\section{Resultados}

Los principales mercados internacionales en el año 2017 de las empresas agroexportadoras de Junín, fueron Estados Unidos, Holanda, Francia, China, Bélgica, Ecuador, Alemania y España. También, al cierre del año, el monto de las agroexportaciones alcanzaron los FOB US\$ 110.75 millones (Tabla 1), siendo este un gran avance para este sector. Este panorama se debe en parte al apoyo que el Estado ha brindado a las agroexportadoras en temas de innovación; es así como, para el año de estudio, la innovación en el producto cacao alcanzó los S/ . 1.02 millones; mientras que la innovación en café llegó a los $\mathrm{S} /$. 2.59 millones y; respecto a la palta y otras frutas fue de S/ 8.99 millones (Tabla 1).

Tabla 1

Mercados para las empresas agroexportadoras de la región Junín, 2017

\begin{tabular}{lcc}
\hline \multicolumn{1}{c}{ Mercado } & $\begin{array}{c}\text { Número } \\
\text { de empresas }\end{array}$ & $\begin{array}{c}\text { FOB } \\
\text { (US\$ miles) }\end{array}$ \\
\hline Estados Unidos & 72 & $35,946.83$ \\
Países Bajos (Holanda) & 35 & $20,616.09$ \\
Francia & 14 & $15,775.52$ \\
China & 6 & $5,523.02$ \\
Bélgica & 12 & $5,446.58$ \\
Ecuador & 10 & $4,078.03$ \\
Alemania & 22 & $3,901.74$ \\
España & 20 & $3,704.98$ \\
Australia & 7 & $2,442.61$ \\
Canadá & 34 & $1,755.28$ \\
Reino Unido & 10 & $1,534.30$ \\
Italia & 19 & $1,392.90$ \\
Países Bajos & 11 & $1,042.64$ \\
Otros países & 126 & $7,593.12$ \\
$\quad$ Total & 398 & $110,753.65$ \\
\hline
\end{tabular}

Nota: Tomado de PromPerú (2017)

El desarrollo del mercado y el apoyo del Estado ha permitido que las empresas agroexportadoras de la región Junín, desarrollen alguno de los tipos de innovación de acuerdo a la clasificación del Manual de Oslo. 
En base a los tipos de innovación de mercado, se ha procedido a estimar el modelo econométrico de la "ecuación (1)" que expresa el nivel de exportaciones frente a los tipos de innovaciones realizadas en las empresas agroexportadoras durante el año 2017.

El modelo econométrico final, entre el logaritmo de las exportaciones y las variables X1, X2, y X4, muestran que las probabilidades de $0.0013,0.0013$ y 0.0244 , respectivamente, siendo todos significativos, además de presentar un $\mathrm{R}$-Cuadrado $=0.800$, siendo un modelo que cumple con las condiciones y resulta ser explicativo (Tabla 2). Asimismo, los resultados muestran que los tipos de innovación, tales como de productos, de procesos y de mercado, fueron significativos al momento de explicar el nivel de exportaciones de las empresas agroexportadoras de la región Junín.

Tabla 2

Modelo de exportaciones e innovación en las empresas agroexportadoras de Junín

\begin{tabular}{ccc}
\hline Variable dependiente: & \multicolumn{2}{c}{ Log(export) } \\
\hline N & \multicolumn{2}{c}{36} \\
\hline Variable & $\begin{array}{c}\text { Coeficiente } \\
\text { (E.E.) }\end{array}$ & Significancia \\
\hline Innov. Producto $\left(\mathrm{X}_{1}\right)$ & 0.1784 & $* *$ \\
& $(0.0508)$ & \\
Innov. Procesos $\left(\mathrm{X}_{2}\right)$ & 0.3513 & $* *$ \\
Innov. Mercado $\left(\mathrm{X}_{4}\right)$ & $(0.0993)$ & $*$ \\
Constante $\left(\beta_{0}\right)$ & 0.9342 & $* *$ \\
R-Cuadrado & $(0.3954)$ & \\
F-stat & 11.0323 & \\
CIA & $(0.3687)$ & \\
\hline
\end{tabular}

$\log ($ export $)=$ logaritmo natural de las exportaciones; $\mathrm{N}=$ muestra; E.E. = error estándar; CIA = Criterios de Información de Akaike; F-stat $=$ Estadístico F; Innov = Innovación; $* *=$ significativa al $0.01 ; *=$ significativa al 0.05 .

\section{Figura 1}

Programas nacionales (Innovación) según sus ventas en la región Junín. Tomado de PromPerú (2017)

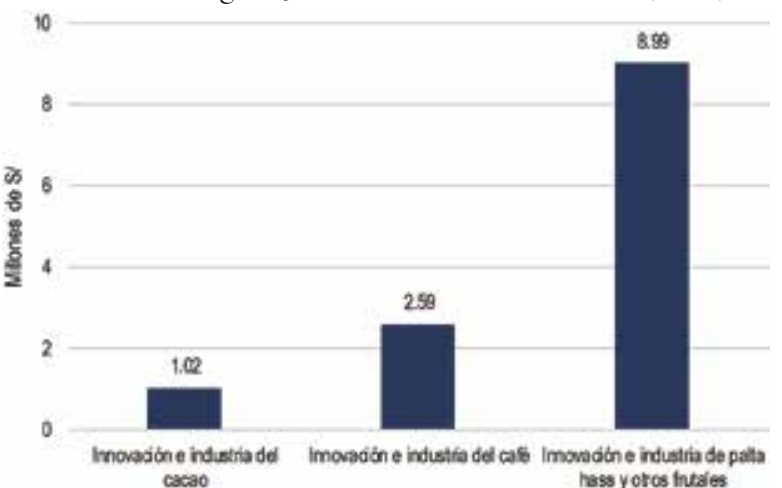

Incluso las innovaciones en productos y procesos fueron altamente significativas (sig. $<0.01$ ). No obstante, la innovación organizativa no resultó ser un variable significativa, esto se debería a que el nivel de exportación no estaría dependiendo del nivel de profesionalización del recurso humano, es muy probable que la experiencia basada en los años de trabajo y estancia en el mercado, sea el principal factor que permite que una empresa agroexportadora se mantenga en el mercado; además, si una empresa tiene una buena organización, resulta también que será capaz de innovar en productos, procesos y mercado, por lo que estaría siendo redundante, el cual fue verificado mediante una prueba de variable redundante

En general, se ha obtenido que la innovación en las empresas agroexportadoras, sí influye en las ventas de las exportaciones totales de las empresas en estudio. En el caso de la innovación de productos, incrementar una nueva presentación de productos, manteniendo constante los demás tipos de innovación, se traduce en un incremento de $17.8 \%$ en el nivel exportaciones. Respecto a la innovación de procesos, contar con una certificación adicional aumenta las exportaciones en $35.1 \%$; es preciso señalar que los países demandantes tienen sus propias condiciones, por lo que contar con más certificaciones en distintos países expande la demanda de los productos, de manera que se exporte en mayor nivel. Respecto a la innovación de mercado, aplicar publicidad vía página web, permite que las exportaciones puedan incrementarse hasta en un $93 \%$; es necesario tener en cuenta que, presentar los productos agrícolas vía un portal ofrece apertura hacia distintos mercados, del total empresas agroexportadoras, solo 16 cuentan con una página web. Es importante tener en cuenta que el incremento marginal es alto, pues las empresas agroexportadoras están en etapa de crecimiento, es así que se observa una tasa alta con respecto a la variable de innovación de mercado. Por lo tanto, es importante que las empresas agroexportadoras innoven en distintas formas, de manera que, puedan acceder a mercados y así incrementar su nivel de exportaciones.

\section{Discusión}

Creamer y Amaria (2012), corroboraron la importancia del conocimiento y aprendizaje organizacional en los mercados externos, donde la competencia resulta ser más fuerte y se requieren de un alto nivel de innovación, asumiendo que la elevada propensión exportadora incrementa las posibilidades del gerente en relación a la innovación. Asimismo, Freiling (1998), establecía que efectivamente el desempeño de la innovación a través del incremento en investigación, desarrollo e innovación dependen de la relación con el cliente, la estabilidad laboral y la formación profesional de los trabajadores de las Pymes mexicanas dedicadas a la exportación. Por otra parte, Schumpeter (1997), señalaba que el desarro- 
llo económico depende de la capacidad de innovar en todos los aspectos y sectores, a través de un proceso dinámico en el cual la nueva tecnología sustituya a la antigua. Se corrobora el modelo de la innovación basado en el Manual de Oslo. Bilkey y Tesar (Bilkey \& Tesar, 1977), señalaron que el modelo entre la innovación e internacionalización radica en la asimilación de una empresa en la decisión de elevar su compromiso internacional, para ello es necesario realizar innovaciones en un sentido amplio y complejo, siendo las decisiones de innovación e internacionalización determinaciones deliberadas y originales.

La implicancia primordial está en identificar a la innovación como el pilar fundamental dentro de una organización y, más aún, en una empresa dedicada al comercio exterior, pues la globalización y los avances tecnológicos hacen que el proceso productivo requiera de nuevos modelos y formas de producción, dado que el mercado lo requiere y la empresa en primer lugar, subsista y, en segundo lugar, crezca.

Para lograr que sean competitivas en el mercado, tanto nacional como internacional, las empresas agroexportadoras de la región Junín, requieren estar a la vanguardia en lo referente al proceso productivo del bien, utilizando la tecnología más adecuada que implique obtener productos de calidad a un precio competitivo; además, es necesario que la organización y la promoción de venta sea la adecuada. Es decir, las empresas agroexportadoras deben de innovar para mejorar su desempeño en el mercado internacional.

\section{Conclusiones}

- El desarrollo del mercado y el apoyo del Estado ha permitido que las empresas agroexportadoras de la región Junín, desarrollen alguno de los tipos de innovación de acuerdo a la clasificación del Manual de Oslo.

- La innovación en las empresas agroexportadoras, sí influye en las ventas de las exportaciones totales de las empresas en estudio.

\section{Referencias bibliográficas}

Albizu, E., Olazaran, M., Otero, B., \& Lavía, C. (2012). Innovación en las pymes industriales: una visión desde el modelo interactivo. Revista Internacional de Organizaciones, 0(7), 17. https://doi.org/10.17345/ rio7.17-43

Anzola, P., Bayona Sáez, C., \& García-Marco, T. (2015). Universia Business Review. Universia Business Review, ISSN 1698-5117, No. 46, 2015, págs. 70-93. [publisher not identified]. Retrieved from https://dialnet.unirioja.es/servlet/ articulo?codigo $=6284687$
Avendaño-Ruiz, B. D. (2017). Innovaciones tecnológicas en el sector hortícola del noroeste de México: rapidez de adopción y análisis de redes de difusión. Corpoica Ciencia y Tecnología Agropecuaria, 18(3), 4. https://doi.org/10.21930/rcta.vol18_ num3_art:740

Barrientos-Fuentes, J. C., \& Berg, E. (2013). Impact assessment of agricultural innovations: a review. Agronomía Colombiana, 31(1), 120-130. Retrieved from http://www.scielo.org.co/scielo.php?scrip$\mathrm{t}=$ sci_abstract\&pid=S0120-99652013000100015

Berk, A. (2018). Factors affecting the exit from farming of young farmers in Turkey: the case of Niğde province. Ciência Rural, 48(8). https://doi.org/10.1590/0103-8478cr20180471

Bilkey, W. J., \& Tesar, G. (1977). The Export Behavior of Smaller-Sized Wisconsin Manufacturing Firms. Journal of International Business Studies, 8(1), 93-98. https://doi.org/10.1057/palgrave.jibs. 8490783

Bin, A., Gianoni, C. M., Vieira Mendes, P. J., Rio, C. T., Monteiro Salles-Filho, S. L., \& Capanema, L. M. (2013). Organization of research and innovation: a comparative study of public agricultural research institutions. Journal of Technology Management \&Innovation, 8, 95-96. https://doi.org/10.4067/ S0718-27242013000300048

Bin, A., \& Salles-Filhoa, S. (2012). Science, technology and innovation management: contributions to a methodological framework. Journal of Technology Management \& Innovation, 7(2), 73-86. https:// doi.org/10.4067/S0718-27242012000200007

Carvalho, L., Avellar, A. P. M. de, Carvalho, L., \& Avellar, A. P. M. de. (2017). Innovation and productivity: empirical evidence for Brazilian industrial enterprises. Revista de Administração, 52(2), 134-147. https: / / doi.org/10.1016/j.rausp.2016.12.009

Chávez, R. X., Lombeida, E. D., Pazmiño, Á. M., \& Flora del C, V. (2015). Innovation in the agricultural sector: experiences in Latin America. Ciencia e Investigación Agraria, 42(3), 16-16. https://doi. org/10.4067/S0718-16202015000300016

Creamer, W., \& Amaria, P. (2012). The effect of business transformation and innovation economics on sustainable corporate competitive advantage. Research in Business \& Economics Journal, 1-35. https: // doi. org/10.1016/j.clinph.2015.04.072

Enjolras, M., Camargo, M., \& Schmitt, C. (2016). SMEs' innovation and export capabilities: identification and characterization of a common space using data spatialization. Journal of Technology 
Management \& Innovation, 11(2), 56-69. https: // doi.org/10.4067/S0718-27242016000200006

Fadhil, R., Syamsul Maarif, M., Bantacut, T., \& Hermawan, A. (2017). Assessment of innovation potential of gayo coffee agroindustry. Quality Innovation Prosperity, 21(3), 114-126. https://doi. org/10.12776/QIP.V21I3.888

Flores Leal, P., Del, M., \& Flores, R. S. (2013). El comportamiento innovador en valor agregado del sector agrícola en el Estado de Sinaloa. J. Technol. Manag. Innov (Vol. 8). Retrieved from http://www. jotmi.org

Freiling, J. (1998). Business model innovation: A concept between organizational renewal and industry transformation, 1-6.

Frezatti, F., Bido, D. de S., Cruz, A. P. C. da, \& Machado, M. J. C. (2017). Impacts of interactive and diagnostic control system use on the innovation process. BAR - Brazilian Administration Review, 14(3). https: / / doi.org/10.1590/1807-7692b ar2017160087

Fuentes, R. A., \& Soto, A. R. (2015). Non-technological innovations in Chilean agricultural firms: what motivates the decision to innovate and the propensity of innovation? Ciencia e Investigación Agraria, 42(2), 4 4. https://doi.org/10.4067/S071816202015000200004

Gras, C., \& Hernández, V. (2016). Modelos de desarrollo e innovación tecnológica: una revolución conservadora. Mundo agrario (Vol. 17). Centro de Estudios Histórico-Rurales. Retrieved from http://www.scielo. org.ar / scielo.php?script=sci_arttext\&pi$\mathrm{d}=\mathrm{S} 1515-59942016000300004$

Hernández Mirafuentes, F., \& Santamaría Basulto, F. (2016). Análisis de las redes de financiamiento del Sistema Nacional de Innovación Agropecuaria en México. Revista Mexicana de Ciencias Agrícolas, 5(SPE7), 1297-1301. https://doi.org/10.1038/ nature 02691

Kleinsmann, M., Valkenburg, R., \& Sluijs, J. (2017). Capturing the value of design thinking in different innovation practices. International Journal of Design, 11(2), 25-40. https://doi.org/10.1525/ cond.2008.110.1.80.80

Olaya, A. (2008). Economía de la innovación y del cambio tecnológico: una aproximación teórica desde el pensamiento schumpeteriano. Revista Ciencias Estratégicas, 16(20), 237-246. Retrieved from http://dialnet. unirioja.es/descarga/articulo/2991260. pdf\% 5 Cnhttp: / / w w w.redalyc.org / pdf/1513/151312829002.pdf

Prim, A. L., Amal, M., \& Carvalho, L. (2016). Regional cluster, innovation and export performance: An empirical study. BAR - Brazilian Administration Review, 13(2). https: / / doi.org/10.1590/1807-76 92bar2016160028

PromPerú. (2017). Exportando.pe.

Rosario, F. J. P., Santa Rita, L. P., \& de Albuquerque, P. P. (2013). Technology, relationship and support institutions on sectoral systems of innovation and production in Brazil's northwest bio ethanol and sugar agro-industry. Journal of Technology Management and Innovation, 8, 285-303. https: / / doi.org/10.4067/S071827242013000300054

Sánchez-Mejía, M., \& Gutiérrez-Terán, A. M. (2013). Proceso de construcción del sistema regional de innovación de la biotecnología para la agricultura, la agroindustria y la bioindustria - SRIB en el Valle del Cauca - Colombia. Journal of Technology Management and Innovation, 8(SPL.ISS.3), 260-270. https:// doi.org/10.4067/S0718-27242013000300052

Sánchez, K., Escobar, L., Sánchez, M., \& Concha, G. (2013). Desarrollo local basado en conocimiento e innovación: caso agropolis del Norte. Journal of Technology Management and Innovation, 8, 105-117. https://doi.org/10.4067/S071827242013000300039

Schumpeter, J. (1997). Teoría del desenvolvimiento económico, 1-24.

Scoponi, L., Dias, M. F. P., Pesce, G., Schmidt, M. A., \& Gzain, M. (2016). Cooperación académica en Latinoamérica para la innovación en los agronegocios. Journal of Technology Management and Innovation, 11(2), 111-120. https://doi.org/10.4067/ S0718-27242016000200011

Tello, M. (2017). Innovación y productividad en las empresas de servicios y manufactureras: el caso del Perú. Revista de La CEPAL. Retrieved from https: / / www.cepal.org/es/publicaciones / 41148-innovacion-productividad-empresas-servicios-manufactureras-caso-peru

Turiján Altamirano, T., Damián Huato, M. Á., Ramírez Valverde, B., Juárez Sánchez, J. P., \& Estrella Chulín, N. (2012). Traditional management and technological innovation of maize in San José Chiapa, Puebla. Revista Mexicana de Ciencias Agrícolas, 3(6), 1085-1100. Retrieved from http:// 
www.scielo.org.mx/scielo.php? script $=$ sci_arttext\&pid=S2007-09342012000600003

Vargas-Canales, J. M., Palacios-Rangel, M. I., Camacho-Vera, J. H. , Aguilar-Ávila, J., \& Ocampo-Ledesma, J. G. (2015). Factores de innovación en agricultura protegida en la región de Tulancingo, México. Revista Mexicana de Ciencias Pecuarias, 6(4), 827-840. Retrieved from http:// www.scielo.org.mx/scielo.php?script $=$ sci_arttext\&pid $=$ S2007-09342015000400013

Von Mühlen, A. S. R., Muñoz-Gallego, P. A., Favero, S., Souza, C. C. de, Pedrinho, D. R., \& Reis Neto, J. F. dos. (2016). Strategic orientations and cooperation of external agents in the innovation process of rural enterprises. Ciência Rural, 46(10), 1878-1884. https://doi.org/10.1590/0103-8478cr20160094 